\title{
An Efficient Method for Multiple Face Detection \& Recognition
}

\author{
Pritthish Chattopadhyay, Priyasha Agarwalla, Vaibhav Sancheti, Srikari Sharma, Swarnalatha P
}

\begin{abstract}
The problem of Face detection and recognition is becoming a challenge due to the wide variety of faces and the complexity of the noises and background of image. In this paper we have used C-sharp and Haar algorithm to detect the face. First in this paper the image is taken with a web-camera, storing it in the database and then once again when the person comes in the frame the name of the person is displayed. This paper is done in C-sharp which was a bit difficult for us to do and we have combined both the face detection and the recognition. The proposed method has good output and a good recognition rate. The limitation of the paper is that it does not display the name of the person above the face. In the future work will be carried on the above said topic. While developing the code some sample codes in python but those were basic programs. So this paper aims to find a solution for it and developed in C-sharp. In finding the XML file of haarcascade frontal face detection we found some problems and had to do a bit of research in finding it. The code for face detection and face recognition were found in different places and in this paper the codes for the both has been combined and found some difficulty. To overcome the basic programs we have written the code in $C$-sharp and the difficulty which we faced in combining the two codes have been solved. The solution has been successfully implemented and the code is fully running and the output has been successfully achieved.
\end{abstract}

Keywords: haarcascade, frontal face detection, C-sharp , XML

\section{INTRODUCTION}

The human face has a convoluted pattern. Automatically identifying human faces is a difficult yet a significant issue. In a fully automatic human face recognition system, it is the first important step. The inspiration behind this paper is the breadth of possible applications for facial detection. [1]. From common household objects like digital cameras that automatically focus on human faces to security cameras that actually match a face to a person's identity. Webcams are often used as a security measure for locking a personal computer. The webcam's facial recognition technology allows for the computer to be accessible to the user only if it recognizes their face. Cameras can also use this mechanism to trail human faces and keep a count of the number of people in a shot or in a certain location or even coming in through an entryway [3]. Revised Manuscript Received on December, 2019.

* Correspondence Author, ${ }^{1}$ First Author

Pritthish Chattopadhyay ${ }^{1}$, School of Computer Sciences \& Engineering, VIT University, Vellore, India. pritthish123@gmail.com

Priyasha Agarwalla ${ }^{1}$, School of Computer Sciences \& Engineering, VIT University, Vellore, India. priyasha.agarwalla2015@vit.ac.in

Vaibhav Sancheti ${ }^{1}$, School of Computer Sciences \& Engineering, VIT University, Vellore, India. vsancheti26@gmail.com

Srikari Sharma ${ }^{1}$, School of Computer Sciences \& Engineering, VIT University, Vellore, India. gottimukkalasrikari.sharma2015@vit.ac.in

Swarnalatha $\mathbf{P}^{*}$, School of Computer Science \& Engineering, VIT University, Vellore, India. Pswarnalatha@ vit.ac.in
This technology can be further narrowed down to the recognition and tracking of eyes. If the viewer is not looking, this would save power by dimming the screen. For this paper, we used existing algorithm as a basis for face detection and build upon it to create improvements and explore more data[5]. The aim of the paper is to detect faces and recognize the face of the person by checking from the folder of trained faces. In this paper the main motive is to display the name of the person when the face of the person will be detected. This paper also aims to detect multiple faces and display the name of the multiple persons[2]. The objective of the proposed work is the paper is detecting as well as recognizing the face of the person. In this paper HaarCascade_frontalface_default $\mathrm{xml}$ file and then incorporating the file in multiple face recognition file[6].

\section{LITERATURE REVIEW}

The paper on Face Detection by KesavaRao Seerapu and R. Srinivas (2014) proposes they have displayed a neural system based face recognition framework which can perceive even with complex foundations, enlightenment and mess. They have utilized Robust PCA and Modified RBFN to accomplish face recognition. The many-sided quality and dimensionality is diminished by Robust PCA and RBFN gives better speed when contrasted and local neural strategies, so this technique gives better outcome in genuine time frameworks likewise the above framework has scope for facilitate improvement likewise can be executed in different constant face recognition frameworks[8].

Nandita Sethi and Alankrita Aggarwal (2014) in their paper, have exhibited a face following calculation progressively camera input condition. To identify the face in the picture, they have utilized a face locator in light of the Haar-like highlights. This face locator is quick and hearty to any light condition. For highlight focuses extraction, we have utilized the calculation of Shi and Tomasi to extricate include focuses. This technique gives great outcomes. To track the facial element focuses, Pyramidal Lucas-Kanade Feature Tracker KLT calculation is utilized. Utilizing identified focuses with the calculation of Shi and Tomasi, they have great outcomes in video grouping and continuously procurement. They got comes about show that the proposed calculation can precisely separate facial highlights focuses. The future work they will incorporate separating include indicates with a few conditions restrict the number of highlight focuses in bouncing box and pick just the focuses which depict well the state of the facial include. The work will be utilized for continuous facial articulation recognition application. Encourage the work can be stretched out by recognizing faces at various slants or inclines as Haar classifier has its own restrictions[9].

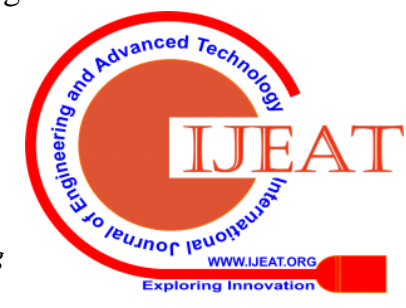




\section{An Efficient Method for Multiple Face Detection \& Recognition}

Shang-Hung Lin (2000) proposes Face recognition is a both testing and critical recognition method. Among all the biometric strategies, face recognition approach has one awesome favorable position, which is its ease of use (or non-nosiness). In the paper, he has given an initial study for the face recognition innovation. He has secured issues, for example, the bland structure for face recognition; factors that may influence the execution of the recognizer, and a few cutting edge face recognition calculations. He trusts this paper can give the per users a superior comprehension about face recognition, and we empower the per users who are occupied with this subject to go to the references for more itemized examination[10].

Anil K Jain (2004) proposes the examination has made commitments to face identification and recognition. Current ways to deal with face recognition are for the most part in view of $2 \mathrm{D}$ power pictures. $2 \mathrm{D}$ pictures are not invariant to changes in enlightenment; facial posture, facial adornments, and appearance, bringing about poor face recognition execution. They have created calculations that conquer huge numbers of these confinements by consolidating data from distinctive calculations, using a bland morphable 3D face model and building careful 3D models from a laser scanner[11].

Ivanov et al(2014) In our past face recognition framework facial segments were removed from the info picture and consolidated into a single element vector which was then sustained into the recognition classifier. As an option, we proposed to prepare recognition classifiers on each of the parts independently and at that point consolidate their yields. Three well known methodologies for consolidating the yields have been assessed: voting, total of yields, and result of yields. We additionally proposed another strategy in light of the dissemination of the experimental blunder for weighting the yields before their mix. Trials were completed on two informational collections: the first set included for the most part low determination, frontal face pictures of six individuals recorded by an observation camera. The second set comprised of engineered preparing pictures of ten individuals and genuine test pictures with extensive varieties in posture and enlightenment. On the two sets we accomplished a noteworthy change over our past framework. In general, the result of the yields hardly beat the other two blend methodologies. By running our new weighting calculation on $10 \%$ of the test set, we could build the recognition rate by an expansive edge without retraining the part classifiers. Delac et al In this paper, another parallel model for face recognition is proposed. There are three variations of parallel model which join diverse variations of LDA. The proposed using data frame recurrence and spatial areas. The two highlights are handled in parallel way. LDA is hence connected on the highlights to counter high dimensionality issue that experience by include combination strategy. The high acknowledgment rate that is accomplished by the proposed techniques demonstrates that highlights of the two spaces contribute significant data to the framework. Parallel model 1 and 2 gave the best outcome. Parallel model accomplished 99\% and $96.7 \%$ acknowledgement rate in ORL and FERET database separately[12].

Swarnalatha (2013) in the paper assumes an indispensable part in the centrality of the investigation of a picture in

restorative picture preparing field, is picking up thought of numerous specialists in present day times. The acknowledgment of issues introduce in the crushed part of a picture is basic for based field. In this paper, we center at building up an approach for better order of therapeutic pictures. Our technique depends on the idea of a novel fluffy approach with bit plane (FCMBP) calculation. The bit plane sifting strategy is utilized to cut the given picture for grouping to discover the demolished area of the given picture[13].

\section{PROPOSED METHODOLOGY}

In this paper we have download the Haarcascade_frontalface_default $\mathrm{xml}$ file and included the file in the program. This paper has combined both the face detection and face recognition code. The sample code for face detection was available in the internet and the code was written in python. A code in C-sharp language has been written. This paper main aim is to detect the face in front of the web-camera and then store the face of the person at different angles in our database. When that person comes in front of the web camera after sometime or passes through the web-camera his face is detected and the name of the person is displayed to us. This helps in security purpose and easy identification of people. In this paper the idea of an image and are that face detection is applied on it. After the face detection takes place after that feature extraction takes place. Once the feature extraction happens it goes on to the face detection Section in which training happens from the "trained face" folder and evaluation takes place i.e. whether the face matches with the image in the database or not and name of the person is displayed.

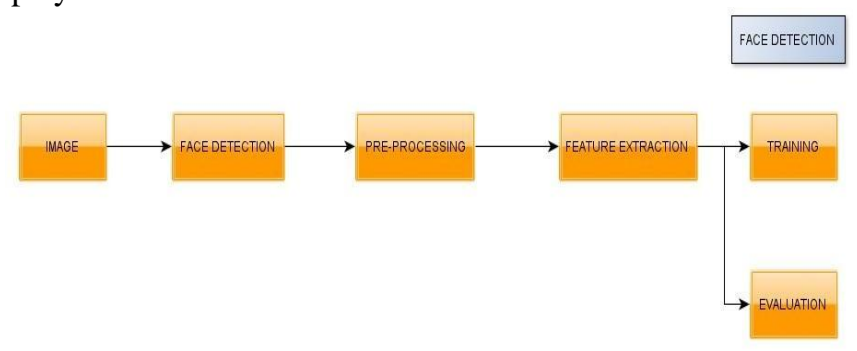

Fig. 1 - Flowchart depicting various steps

\section{SYSTEM ENVIRONMENT}

This section covers requirements related to the expected functionality of the system. Two or more sufficiently different methods for face recognition shall be implemented. A complete implementation of the Haar method shall be provided .A user interface incorporating the different methods and their results shall be implemented. The interface shall provide functionality to select an input image to be recognized .Results from each method shall be displayed clearly and ordered by the closest match Rationale.The interface will allow the methods to be tested and evaluated. The final product detects face with the help of a web-camera and then displays the name of the person who is detected. The Product is efficient and is reliable. This product can be used for security purposes. The product displays the name of the person who is detected and displays name accurately. 
The assumption which is considered in the paper is that the faces will be stored in the folder "trained faces" and then the name is displayed by matching with the face detection. The names stored to be stored before hand and then the recognition takes place. Non-Functional requirements are concerned with aspects of the application such as usability and performance or more generally, aspects not related to the functionality of the system. The product needs to have a working web-camera and the XML file needs to be imported in the main working file. Visual Studio needs to be installed in the system in which the face detection and recognition code is executed. The paper of face detection and recognition is very efficient because it recognizes and displays the name of the person as soon as the person comes in the frame of the web-camera. The paper is reliable as in all the test cases the correct name of the person who is recognized is displayed.

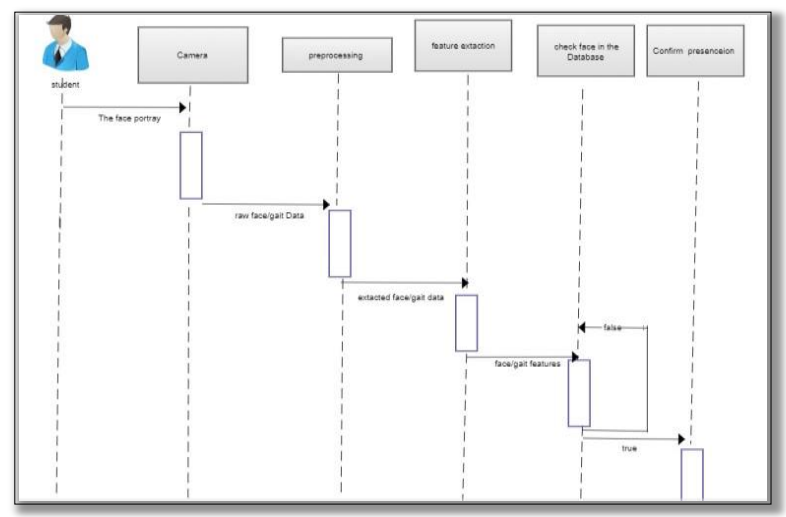

Fig. 2 - System Environment for Face Detection and Recognition

\section{RESULTS \& DISCUSSIONS}

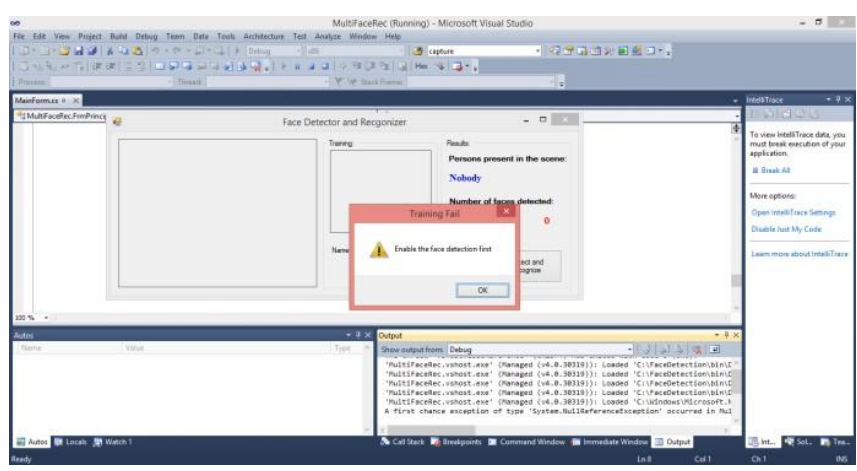

Fig. 3 - Folder showing enable first

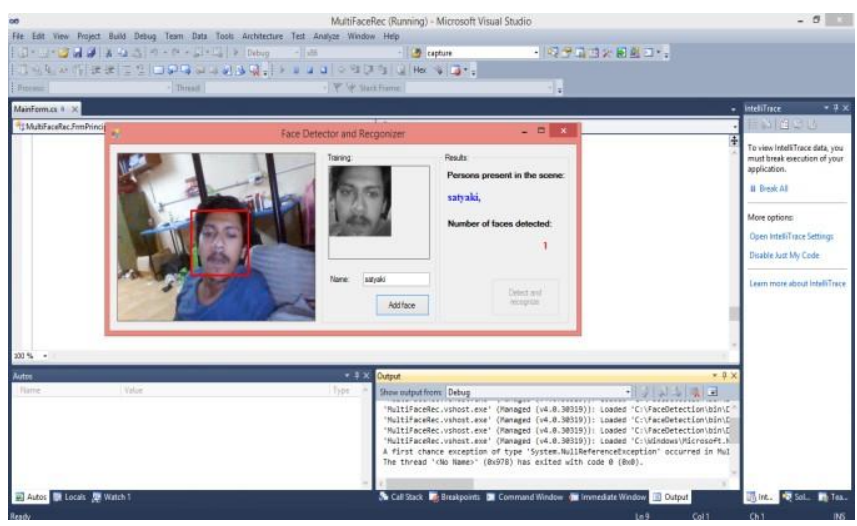

Fig. 4 - Displaying name of Recognized person

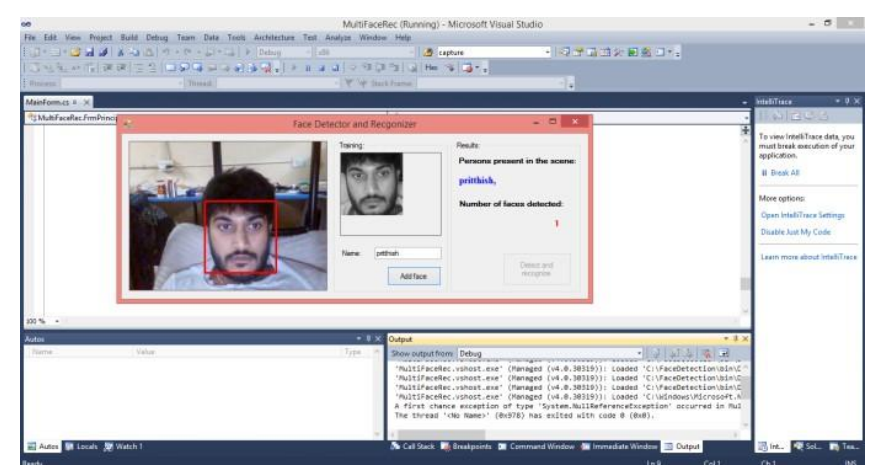

Fig. 5 - Displaying name of Recognized person

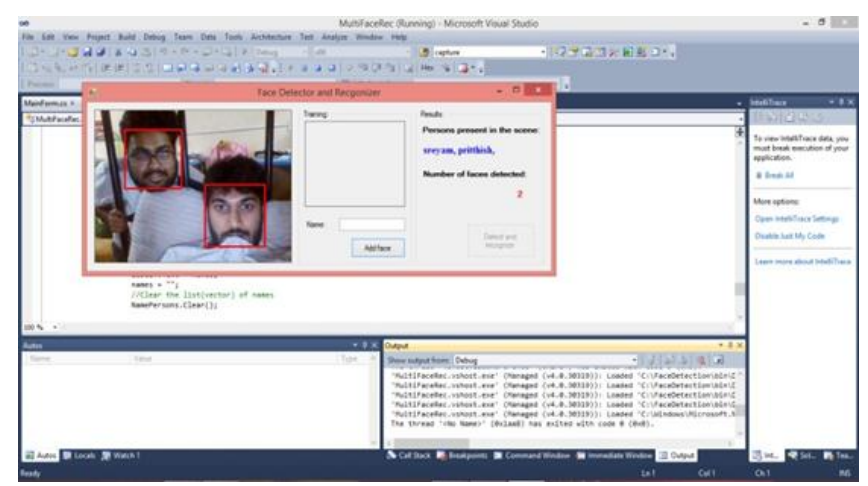

Fig. 6 - Multiple Recognition

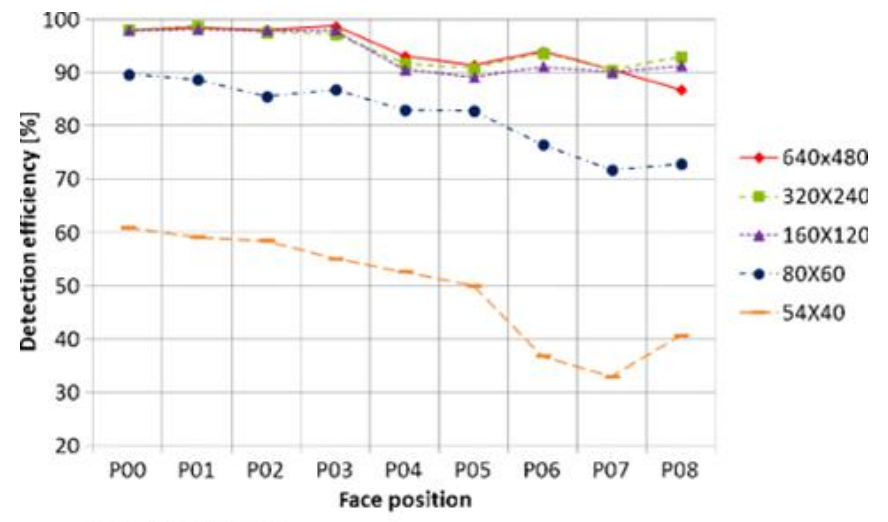

POO - the front face

P01 - head directed slightly upward (25")

P02 - head directed slightly upward $\left(25^{\circ}\right)$ and to the left $\left(20^{\circ}\right)$

P03 - head directed slightly to the left (200)

P04 - head directed slightly down $\left(25^{\circ}\right)$ and to the left $\left(20^{\circ}\right)$

P05 - head directed slightly down (25")

P06 - head directed upward $\left(45^{\circ}\right)$ and to the left $\left(30^{\circ}\right)$

P07 - head directed to the left (45")

P08 - head directed down $\left(45^{\circ}\right)$ and to the left $\left(30^{\circ}\right)$

Fig. 7 - Graph depicting efficiency for various angles of face

Published By: 


\section{An Efficient Method for Multiple Face Detection \& Recognition}

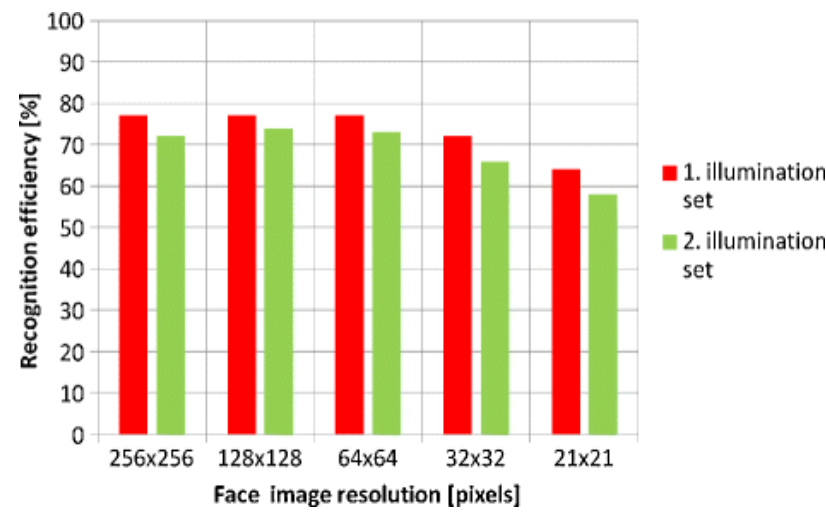

Fig. 8 - Influence of image resolution and various face positions on face detection process.

\section{CONCLUSION}

In this paper is that it recognizes the faces and displays the name of the person. In this paper the work on multiple detection of faces of the persons and it displays both the name of the persons simultaneously. In the above test cases the samples have given all the samples in which it displays the names of the persons and multiple recognition is also shown. In this paper a screenshot of a folder named "Trained Faces" is given in which all the faces that are stored is shown.

The code has been written in C-sharp and executed our code. This paper detects the face of the person and detects them and displays the name of the person. An active web-camera must always be there for the detection and recognition process. This paper also displays the names of the multiple persons that are detected. Multiple persons can be recognized and the number of persons in the frame is also displayed which is showed in the expected results. One of the limitations in our paper is that all the files need to be stored in the system in which facial recognition needs to be carried out and all the softwares should be installed beforehand for the execution. It also does not display the name of the person above the face of the person which we have planned out to do in future work. For the future work the name which is displayed at the side of the application we will try to display the name above the name of the person who is detected and try to develop our paper.

\section{REFERENCES}

1. http://www.ijcscn.com/Documents/Volumes/vo2issue5/ijcsen201202 0504.pdf

2. http://www.face-rec.org/journalsbooks/Delac_Grgic_Bartlett_Reent Advances_in_Face_Recognition.pdf

3. http://bheisele.com/fg-04-2.pdf

4. http://www.dtic.mil/dtic/tr/fulltext/u2/a430264.df

5. http://citeseerx.ist.psu.edu/viewdoc/download?doi=10.1.1.208.7800\& rep=rep1\&type $=$ pdf

6. R.Rojas(1996),NeuralNetwork An Introductions,Springer-Verlag, Berlin,-IEEETransactions of Neural Networks. vol.8, no.1,pp158-200

7. http://ieeexplore.ieee.org/abstract/document/652 8524/

8. https://arxiv.org/pdf/1603.01249.pdf

9. https://www.researchgate.net/publication/265269977_Robust_Face Detection_and_Tracking_Using_Pyramidal_Lucas_Kanade_Tracker_ Algorithm

10. https://core.ac.uk/download/pdf/26858086.pdf

11. http://biometrics.cse.msu.edu/Presentations/AnilJain_FaceRecognitio n_KU10.pdf

12. https://ieeexplore.ieee.org/abstract/document/1301569

13. https://www.researchgate.net/publication/325982321_Facial_expressi on_detection_using_facial_expression_model

\section{AUTHORS PROFILE}

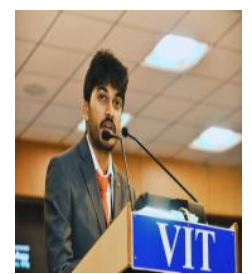

Pritthish Chattopadhyay completed his B-tech in Computer Science and Engineering from Vellore Institute of Technology, Vellore in the year of 2019. He is currently working as an Associate Data Scientist in Novartis Healthcare Private Limited, Hyderabad. He has two papers published on Smart Garbage Monitoring System and Image Compression Using Bayesian Fourier in IJERT journal. He was the Convener of Technical Fest graVITas'18(VIT, Vellore) and also the Genera Secretary of VIT SPARTANS (VIT, Vellore).

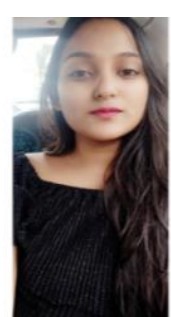

Priyasha Agarwalla completed her B-tech from Vellore Institute of Technology, Vellore in Computer Science and Engineering in the year of 2019. She is currently working as a Software Engineer in HSBC Technology India, Pune. She has participated and secured ranks in various National and International Olympiads. In her college days she was the coordinator of Publicity and Marketing of cultural fest Riviera'17(VIT, Vellore). She was also the Events Head of Creativity Club (VIT, Vellore). She is fond of social service and has been a teacher in each one teach one project.

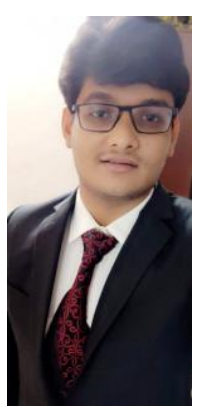

Vaibhav Sancheti just recently graduated (April 2019) from VIT University, Vellore with a Bachelor's of Technology degree in Computer Science. $\mathrm{He}$ is very keen on undertaking research related projects and has a paper published in International Journal of New Technology and Research (IJNTR) on "An Initiative to Improve Quality of Software Testing Techniques and Calculating Total Number of Failures using Bayesian Method". His research interests include Machine Learning, Data mining and image processing using OpenCV. .

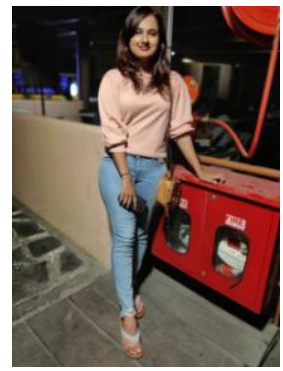

Srikari Sharma just recently graduated (April 2019) from VIT University, Vellore with a Bachelor's of Technology degree in Computer Science and is currently working as a Market Research and Data analyst for Trouw Nutrition Pvt Ltd. She is interested in areas related to data analysis and visualization and has undertaken related projects. She also has a paper published in International Journal of New Technology and Research (IJNTR) on "An Initiative to Improve Quality of Software Testing Techniques and Calculating Total Number of Failures using Bayesian Method" and her another paper " Comparison on YOLO vs SSD" is under review. Her research interests include Visualization, Data mining $\&$ analysis and image processing.

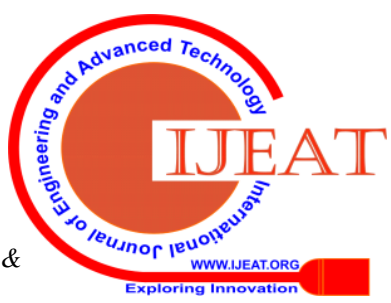




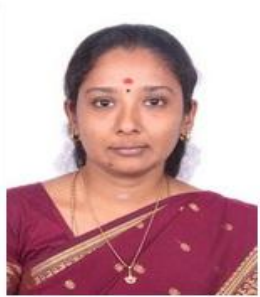

Swarnalatha Purushotham is an Associate Professor, in the School of Computer Science and Engineering, VIT University, at Vellore, India. She pursued her Ph.D. degree in Image Processing and Intelligent Systems. She has published more than 75 papers in International Journals/International Conference Proceedings/National Conferences. She is having $18+$ years of teaching experiences. She is a professional member of IACSIT, CSI, ACM, IACSIT, IEEE (WIE), and ACEEE. She is an Editorial board member/reviewer of International/ National Journals and Conferences. Her current research interest includes Image Processing, Remote Sensing, and Artificial Intelligence, Big data Analytics, Deep Learning and Software Engineering. 